\title{
Strengthening the Foundations Underlying the Enterprise Engineering Manifesto
}

\author{
Martin Op 't Land ${ }^{1,2}$ and João Pombinho ${ }^{3,4}$ \\ ${ }^{1}$ Capgemini Netherlands, P.O. Box 2575, 3500 GN Utrecht, The Netherlands \\ ${ }^{2}$ Delft University of Technology, Delft, The Netherlands \\ ${ }^{3}$ Instituto Superior Técnico, Technical University of Lisbon, Lisbon, Portugal \\ ${ }^{4}$ CODE - Center for Organizational Design \& Engineering, INESC INOV, \\ Rua Alves Redol 9, Lisbon, Portugal \\ martin.optland@capgemini.com, jpombinho@acm.org
}

\begin{abstract}
The discipline of Enterprise Engineering aims for enterprises to operate as a unified and integrated whole. This discipline therefore adopts the mission to develop theories, models, methods and other artifacts for the analysis, design, implementation and governance of enterprises in a theoretically rigorous and practically relevant manner. The Enterprise Engineering Manifesto postulates the dualities of concepts function/construction perspective, blackbox/white-box models and subjective/objective as being opposed to each other in one-on-one relationships.

Illustrated by the Pizzeria case, it becomes clear (a) that functions can be defined objectively, and (b) that a valuation perspective should be added that truly focuses on the relationship between a system and its stakeholders. These insights can support building stronger bridges between management and organization sciences - traditionally stronger in functional approaches - and information systems science, and computer science - traditionally stronger in constructional approaches.
\end{abstract}

Keywords: Enterprise Engineering, Enterprise Engineering Manifesto, Function perspective, Construction perspective, Black-box models, White-box models.

\section{Introduction}

According to the Enterprise ${ }^{1}$ Engineering Manifesto (EEM)[1], Enterprise Engineering is an emerging discipline that deals with developing theories, models, methods and other artifacts for the analysis, design, implementation and governance of enterprises in a theoretically rigorous and practically relevant manner. Since this goal encompasses different domains of knowledge, enterprise engineering is itself a multidisciplinary discipline that combines concepts from management and organization science, information systems science, and computer science. One of the challenges

\footnotetext{
${ }^{1}$ We use the term "enterprise" for any goal-oriented cooperative of people.

A. Albani, D. Aveiro, and J. Barjis (Eds.): EEWC 2012, LNBIP 110, pp. 1-14, 2012.

(C) Springer-Verlag Berlin Heidelberg 2012
}

The original publication is available at www.springerlink.com 
that arise from this approach is being able to coherently and consistently combine the various components of an enterprise, while coping with the adaption and change concerns that inherently will arise over time. Indeed, the complexity of businesses and the high change pace of their environments, coupled with increasing ICT support, turn the gap between strategy and its implementation into a major challenge. Studies indicate as much as 90 percent of organizations fail to succeed in applying their strategies [2].

The EEM [1] states that management and organization sciences address these issues predominantly from a functional perspective. Conversely, information systems science and computer science mainly make use of the constructional or engineering perspective. The EEM then focuses on the contributions of constructional thinking to the Enterprise Engineering (EE) discipline. Particularly, its Postulate 3 reads:

"There are two distinct perspectives on enterprises (as on all systems): function and construction. All other perspectives are a subdivision of one of these. Accordingly, there are two distinct kinds of models: black-box models and white-box models. White-box models are objective; they regard the construction of a system. Blackbox models are subjective; they regard a function of a system. Function is not a system property but a relationship between the system and some stakeholder(s). Both perspectives are needed for developing enterprises."

Three dualities of concepts are mentioned, and set in one-to-one relationships:

- Function and Construction perspectives;

- Black-box and White-box models;

- Subjectivity and Objectivity.

We agree with EEM that these concepts have a very important role as a referential for the entire EE discipline. Therefore it would be beneficial to clarify their definitions and relations, to not only distinguish these notions, but also be able to connect them. Suppose, for instance, that it would be possible to define functions (more) objectively, then it would be possible to simulate, test and maybe even prove coherence, sufficiency, etc. of a certain construction and implementation to bring about certain functions. This would contribute to a core value of Enterprise Engineering, namely unified and holistic design, truly combining management and engineering disciplines.

The remainder of this paper is structured as follows. Section 2 begins with a brief Problem Statement, and the introduction of the running Pizzeria example for our conceptual exploration. Then Section 3 presents the three mentioned dualities of concepts, instantiated with the Pizzeria example. Next, the three dualities are discussed and compared in Section 4. The paper closes with conclusions and recommendations regarding Enterprise Engineering in general, and regarding the EEM specifically.

\section{Problem Statement}

The main question we want to answer is:

"Looking at the three dualities of concepts - function versus construction perspective, black-box versus white box models, and subjectivity versus objectivity - are these three dualities of concepts really one-on-one?" 
As a first illustration, look at the work of a sluice operator. Without knowing his work instructions (white-box), we can observe his behavior (black-box) and compare that with expected behavior in parameters well known in product and reliability engineering, such as service times, delivery times, Mean Time Between Failure and error rates. Such Quality of Service (QoS) parameters can be expressed and also measured quite objectively - which is a regular practice in Service Level Management, and also the basis for Service Level Agreements (SLA's). So there seems not necessarily to be a problem to define functions objectively.

Comparable examples can be found in the Six Sigma, which uses black-box observations to systematically search for statistical correlations - which could point to causality in (until now may be unknown) white boxes. Also here functional parameters are defined and measured objectively.

Yet another example is given by Dietz [3], looking at the heart as a biological system. The heart has the function of pumping blood, i.e. transporting blood from location A to location B with a certain velocity range and a certain capacity for adapting to fluctuations in viscosity. This function can be expressed objectively and measurably. Dietz then continues to clearly distinguish between function and purpose, where "purpose is a relationship of a system with its stakeholder". May be that is more a place where subjectivity could be expected, since the interests of me in my heart could well differ from those of the heart surgeon, or those of the poor colleague patient waiting for a heart transplant.

We want to approach this -rather conceptual- question with the strategy "validation by instantiation". To instantiate the three dualities of concepts in the next sections, we will use the following adapted version of the classic Pizzeria example from [4], familiar to the EE community.

Pizzeria Case. The Pizzeria is a business organization that provides pizza to its customers $\left(1,000\right.$ pizzas of at least $95^{\circ} \mathrm{C}$ a week with a Mean Time Between Failure of one month). Besides the customers, there are many stakeholders: the investors, the city who grants licenses in return for license fee and the employees working in the pizzeria. It is noteworthy that the customers belong to two distinct segments: regular end-customers and cafés located in luxurious terraces nearby, which complement the offer to their customers with excellent fresh pizza.

In an earlier version of the Pizzeria organization, customers could just walk in and make their wishes known at the counter or could order by telephone. In both cases they had to take away the pizzas themselves. Later, an important new service was introduced: one could have the pizzas delivered to an address. Both means of pizza delivery to the customer co-exist today.

Customers announce themselves at the counter of the pizzeria or make a telephone call. After making her selection from a menu, the customer is informed about the price and the expected time that the order will be ready.

The order is then passed to the kitchen, where the baker prepares the pizzas. Once ready, the pizzas that make up an order are boxed. Depending on the delivery selection, the boxes are then handed over to the customer against payment or delivered a customer-specified address by a transporter, whom also collects the money on behalf of the pizzeria. 


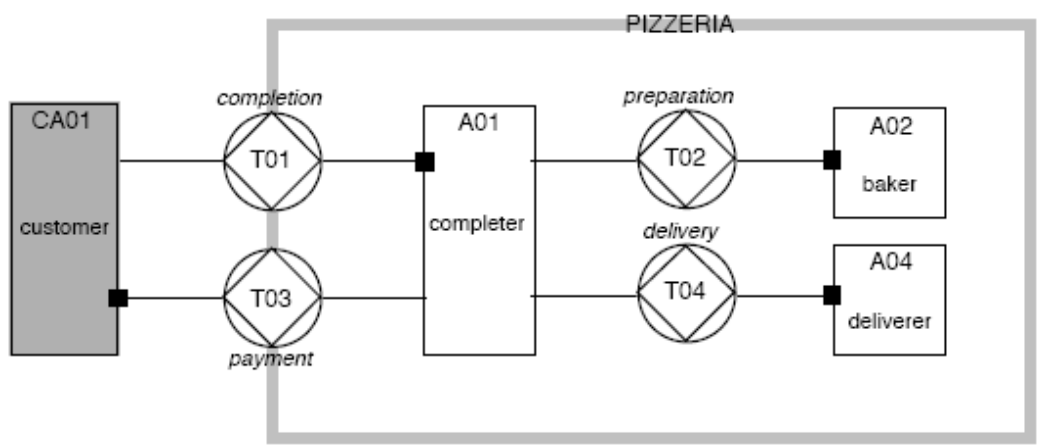

Fig. 1. Detailed Actor Transaction Diagram (according to DEMO) from the Pizzeria [3]

\section{$3 \quad$ Analysis of the Dualities of Concepts}

First we will introduce the Enterprise Engineering concepts of system, model and Generic System Development Process (GSDP). Then we will present each duality and instantiate it with the Pizzeria example.

\subsection{Base Theory: System, Model and GSDP}

System and Model. Dietz [3] defines something as a system if and only if it has: composition - a set of elements of some category (grey nodes in Fig. 2); environment - a set of elements of the same category, disjoint from the composition (white nodes); production - things produced by elements in the composition and delivered to the environment; structure - a set of influence bonds among the elements in the composition, and between them and the elements in the environment (connections between white and grey nodes). Together, these properties are called the construction of a system.

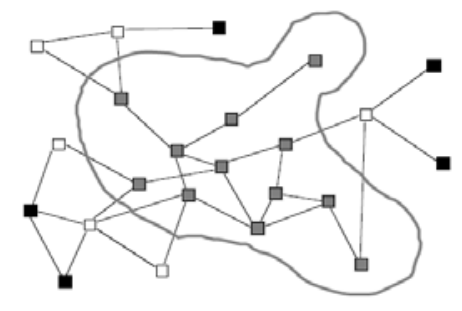

Fig. 2. The construction of a System [3]

Apostel [5] defines the concept of model of a system is as a role as follows:

"Any subject using a system A that is neither directly nor indirectly interacting with a system $B$, to obtain information about the system $B$, is using $A$ as a model for B." 
General System Development Process. In every design process there are two systems involved, the using system (US) and the object system (OS), as depicted in Fig. 3. The OS is the system to be designed; the US is the system that will use the functions or services offered by the OS once it is operational.

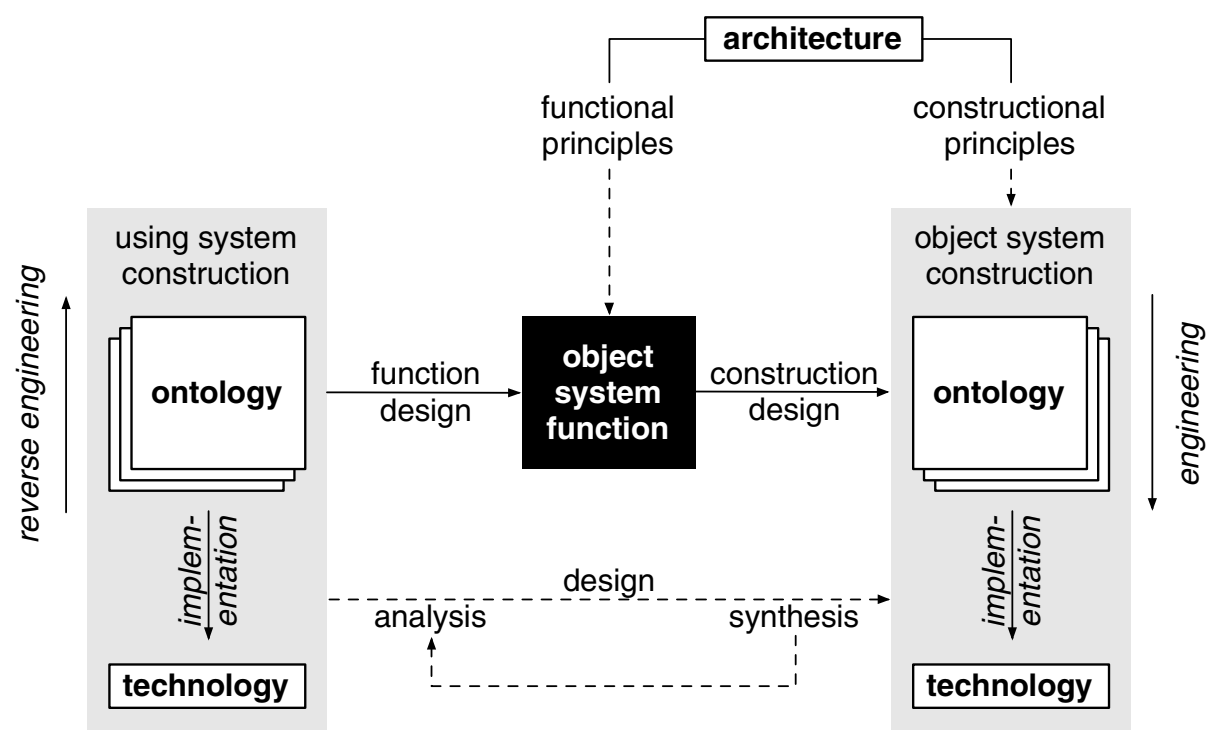

Fig. 3. Generic System Development Process [6]

Function design, the first phase in the design of the OS, starts from the construction of the US and ends with the function of the OS. Function design delivers the requirements of the OS, so a black-box model of the OS. This black-box model clarifies the behavior of the OS in terms of (functional) relationships between input and output of the OS. This function model of the OS does not contain any information about the construction of the OS. Construction design, the second phase in the design of the OS, starts with the specified function of the OS and it ends with the construction of the OS. Construction design bridges the mental gap between function and construction, which means establishing a correspondence between systems of different categories: the category of the US (where the function of the OS is defined) and the category of the OS. Construction design delivers an ontology, the highest level white-box model of the OS. This white-box model clarifies the internal construction and operation of the system in terms of collaboration between its elements to deliver products to its environment. By an ontology or ontological model of a system we understand a model of its construction that is completely independent of the way in which it is realized and implemented. The engineering of a system is the process in which a number of white-box models are produced, such that every model is fully 
derivable from the previous one and the available specifications. Engineering starts from the ontological model, produces a set of subsequently more detailed white-box models and ends with the implementation model. By implementation is understood the assignment of technological means to the elements in the implementation model, so that the system can be put into operation. By technology we understand the technological means by which a system is implemented. A wide range of technological means is available, including human beings and organizational entities, ICT artifacts (e.g., phone, email, computer programs) and mechanical means. In general, the design freedom of designers is undesirable large. Dietz and Hoogervorst [6] therefore define architecture (1) conceptually as a normative restriction of design freedom and (2) operationally as a consistent and coherent set of design principles that embody general requirements, where these general requirements hold for a class of systems. Those principles can be functional or constructional, i.e. restricting the function resp. the construction design of a system.

\subsection{Objectivity and Subjectivity}

The notions of objectivity and subjectivity motivate a long ranging philosophical debate where the quest for truth has a main role.

A proposition is considered objectively true when its truth conditions are met and are not dependent on the mind of the observer. Objectivism, in this context, is an alternative name for realism, the view that there is a mind-independent reality, which implies a definition of truth.

Foundationalism, the notion that knowledge is built upon an unchangeable foundation, would be the only assurance that truth can be achieved. However, the notion that absolutely secure and final knowledge is attainable - the quest for absolute truth was progressively regarded as unfruitful as, even if it is reachable, there does not seem to be a way of being certain about it [7]. Additionally, regarding scientific knowledge, the definition of objectivity states that descriptions of physical phenomena must not change when the phenomena are observed by different observers, under different conditions. Kuhn [8] notably defended the impossibility of scientific understanding being truly objective, due to the nature of scientific organization around community-bound paradigms. No smooth transactions occur between them, Kuhn observed, as a reigning paradigm would only give way to another after overwhelming evidence of unfitness was accepted by most of its supporting community - revolution, rather than evolution. These facts, illustrative of the towards objectivity, should not be met by considering that everything must then subjective, as these are related but different matters - is a voyage, not a destination.

The question is then, what obstacles exist on the way of objectivity? For instance, it seems clear that observations are more likely to be recognized as higher in objectivity when they are simpler and closer to sensory experiences - thus, not so influenced by rational paradigms and viewpoints. This is a debatable point, since artificially comfortable notions such as sensatory experiences and unanimity in observations cannot be taken as necessarily objective - the fact that an audience agrees that a rabbit 
came out of a magician's hat does not make in an objective truth. In this respect, different types of objectivity exist - quantitative versus qualitative. We must also take care to differentiate group consensus as potentially illusory and not any better at providing truth than other approaches, such as statistical observation or scientific inquiry.

Even influenced by paradigms, efforts should be taken to remove that bias in favor of objective. Objectivity is a valued quality in goal-setting, for instance. The well known "SMART" criteria (Specific, Measurable, Attainable, Relevant, and TimeBased) [9], are a good example of striving for objectivity. The distinction between the process of inquiry for truth and its outcomes is also crucial. While no guarantee of absolute certainty on the results of the inquiries can be provided, the meeting of procedural standards must be valued - making some types of inquiries comparatively better than others. Opening to challenge and scrutiny by a critical community is instrumental in freeing from biases as concealed valuations. This is notion is crucial to Popper's Critical rationalism [10], where the quest for continuous falsification follows from the need that a theory has to be falsifiable in order to be scientific.

Concluding, objectiveness depends on the seriousness in the pursuit of criticism and refutation. Full objectivity is not attainable - it is, then, a relative measure of the effort to be objective. Therefore, there are no objective results recognizable as an undisputable truth, only a relatively objective way of working.

Example. In the Pizzeria case, a claim that a specific pizzeria's pizza is the tastiest in town is highly subjective.

Explanation. The fact that different individuals can experience it at different times and in different personal contexts clearly discourages a quest for absolute truth. However, a customer survey can be regarded as a means of increasing objectivity. For instance, its objectiveness can be increased if it is performed by an independent entity, rather than the pizzeria itself, and the criteria are known by all participants.

\subsection{Function and Construction}

The relation between function and construction is defined in [3] by positioning them in the context of a system development process - the Generic System Development Process (GSDP) as presented in section 3.1. Two notions of a system are clearly separated: the teleological, concerned with behavior and function, and ontological, concerned with the system's construction and operation.

Dietz claims[4] that functional models, typically used in disciplines such as management and organizational science, are useful for the purpose of using or controlling a system and inadequate for bringing about changes. There seems to be an implicit implication that these models are fuzzy by nature and unapproachable from a formal perspective that is useful in enterprise engineering. Building on the previous sections, we argue this is not the case, and that functional models can be SMARTly (as explained in the previous section) and even formally defined. 
As presented in the GSDP, a given construction is made to support a function. The function is, then, dependent on construction. According to a survey by Sprint [11], nearly two-thirds of the respondents appeared to have used the backlight of their mobile phones to look for something in the dark. If, for instance, a mobile phone interface is someday replaced by using brainwaves operation instead of hardware, their users will have to use other devices to look for keyholes.

Differentiating Function, Purpose and Value. The function is a characteristic of system and comparatively objective regarding purpose and value (see section on objective/subjective). The first specifies the system's relation with elements on its environment, in terms of inputs and outputs. The second, purpose, specifies what usage is given to the system's function, from a specific external actor's point of view. Value emerges from the relation: it is attributed to a system function by a stakeholder in regard to a specific purpose. Even so, it can be made less subjective and, therefore, relatively freed from bias, namely by 1) specifying the external actor itself in terms of function and construction, and 2) specifying alternative functions that can be used to the same purpose. As an example, the value that the Pizzeria pizza-for-money function has for the caffé is compared against microwaving pre-baked pizzas, lacking freshness and overall quality, and resulting in less value transferable to the endcustomer. As can be noted, the purpose definition process is recursive and results in the notion of purpose being tightly connected to the one of chain. The recursive notion of purpose just presented conceptually extends the basic relation of two elements to an arbitrary number and, in the process, building the purpose structure. The specification is out of this paper's scope, but the point must be made that it is important to segregate purpose-oriented notions from function definitions as they have a different nature and positioning regarding a system and its environment.

Innovation. The relation between the concepts of function and construction is prescribed in the GSDP having construction as subordinate to function - this relation is a consequence of the development process. This follows from the sequence of the process and does not change by considering iteration, since the creative step of the process always happens from function to construction. This also hinders creative reuse of a construction (innovation) because the OS value is limited, by process design, to satisfying the construction of a specific US. Most importantly, the conceptual direction of the process should be reversible; as an application take, for instance, the discovery of new functions for an existing construction.

Example. The way the elements in the Pizzeria construction, namely its delivery (sub) organization, were chosen supports the function of providing pizza to the physical location of customer.

Explanation. It was a strategic decision by the Pizzeria owner to provide pizzas to a given customer location. Also note that the same construction allows providing Pizza 
to both end-customers and business partners - although in this last case some optimizations could be made. It is even the case that the delivery function, by itself, could provide the service to the customers of the business partners - the collection and price visibility would be a little trickier with the current construction, though. Note that in the ontological model the customer types are not differentiated.

\subsection{Black-Box and White-Box}

The general notion of black-box is a metaphor for an abstraction that obscures the internal workings of a given system so that only output, as reaction to some specific input, is observable (left part of Fig. 4). For instance, that the pizzeria is offering 1,000 pizzas of at least $95^{\circ} \mathrm{C}$ a week with a Mean Time Between Failure of one month. Conversely, the composition and structure of a white-box are available for inspection, allowing clarification of the transfer function of the system [12]. While the white-box perspective is embraced by engineering disciplines and objectiveness by need of constructability, the black-box perspective is frequently connected to subjectivity. As we will see, this is not necessarily the case.

Partiality versus Subjectivity. Distinguishing subjectivity from partiality is crucial to clarifying the (wrong) notion of necessary subjectivity of the black-box perspective. The black-box of a system can be partial, in the sense it can represent a limited selection of inputs and outputs. However, these must be correspondent to each other, as in a function's range and domain. Further, and most important, only black-boxes supported by the corresponding white-box are allowed, i.e., the black-box must be coherent with the white-box model.

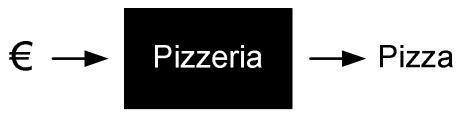

a)

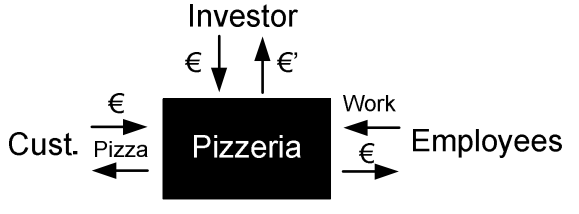

b)

Fig. 4. Pizzeria Black-Box model from a) Customer perspective; and b) Pizzeria perspective

In the example represented in Fig. 4, the Customer can legitimately assume a simplified model of the Pizzeria (a). But using a more complete model with additional stakeholders (b), one must wonder how independent those value exchanges are. Indeed, the holistic and unified approach as ascribed in the EEM must be built upon adequate theory for supporting multiple partial views in an integrated way $-e$ pluribus unum.

Relativity. As with subjectivity and objectivity, presented in the previous section, the concept of relativity is also present regarding black-box and white-box perspectives of 
a system. Indeed, a given systems' white-box may be a black-box (or a set of blackboxes) in another referential of system types. This concept is distinct from a simple aggregation by enforcing the commitment to a specific referential. For instance, take the white-box model of a car, which still holds the chemical system of a battery as a black-box. Following the same reasoning, and having finer thresholds between system types, a given overall system model can be further refined into layers. These layers can be defined to model M-N relations between systems, e.g., the car can use both a battery, solar power and gas as energy providing (sub)systems. Therefore, they end up forming a layered solution path where the uppermost layers are dependent on the lower ones. For instance, this concept is clearly presented regarding DEMO's B, I and D-organizations in [13].

Example. The customer black-box perspective of the Pizzeria is a system that outputs a pizza in exchange for money provided as input (see Fig. 4). From the investor's black-box perspective, the Pizzeria is a system that outputs money (profit) in exchange for input money (investment). From the employee's perspective, the Pizzeria is a system that outputs a/o money (salary) in exchange for input "work".

Explanation. Only elements from the environment and relations that cross the frontier with the composition are relevant from a black-box perspective. A black-box perspective corresponds to a functional vision of the system which, as we have seen, can be partial. However, we must note that the black-box from the Pizzeria's perspective is more than the sum of its parts, in this case more than an aggregation of the individual external perspectives. In this example, the monetary exchanges are not independent as they must be financially balanced.

\section{Discussion}

Regarding the three fundamental dualities analyzed in this paper:

- Subjectivity/Objectivity is the one with a larger application scope as it is independent of a specific research area; it is also the most generally applicable at the philosophical level;

- Function/Construction and Black-box/White-box have one-on-one correspondence; however, they are orthogonal to the Subjectivity/Objectivity duality; moreover, their positioning in the objectivity range does not depend on their fundamental essence but on the effort to make them objective through concept analysis and modeling way of work.

A point must be made regarding relativity and recursivity of their application: a system can be conceived as a series of chained conceptual solutions to problems - it can be argued they always are, at some point in the system's lifecycle as a result from the creative process of problem solving/solution identification. Then, following the relativity principle the black-boxes can indeed be opened to reveal white-boxes, which in turn will again be composed of black-boxes. Since a given construction does not 
support every function, and a particular function is not supported by every construction, logic must be devisable for mutual modeling restriction, either from a positive or negative approach in terms of degrees of freedom.

Coupled with the presented conclusions regarding objectivity, it can be concluded that a chaining of black-box and white-box systems ends with a black-box since there is no complete knowledge of the truth. For instance, take the human body as a set of systems, the muscular system, the molecular physical system and the atomic level of description. There is always a basic theory, which is the recursivity stopping point. A white-box model example such as the model of an atom is also a reference of why it is not necessarily objective; in this case, it is theory-bound. This unassertible objectiveness is also the reason that the concept of usefulness of a model, a very important notion in system design and engineering, must be introduced. In the atomic theory example, while there can be no claim that it corresponds to absolute truth, it has undoubtedly contributed consistently to produce practical results in advancing humanity. In the same way, a theory regarding black-box specification in an integrated way with the construction must be regarded as useful and as sound as possible, provided that demonstrable efforts are made towards objectivity.

A system's construction or function alone is not directly relatable to the usage scenarios it may have: a work of art can be used as a paper weight. Therefore, a different perspective that is subject-sensitive and includes concepts such as purpose, contribution, value and goal is required. Let's designate it, following [14], by valuation perspective. This perspective should 1) differentiate and formally define these concepts; 2) define the relations between them, and 3) define the direct interface with the function perspective and, through it, the indirect interface with the construction perspective.

\section{Conclusions and Recommendations}

\section{$5.1 \quad$ Conclusions}

We found the following weaknesses in the EEM, which we consider worthwhile to consider changing.

Though the EEM says that both the function and the construction perspective are needed for developing enterprises, almost all attention goes to the added value of the construction perspective. Also it is said that the functional perspective "is inadequate for bringing about changes", while it is probably meant that the functional perspective is insufficient for bringing about changes. For the stage of the Manifesto as an activistic document, to correct common misunderstandings in Enterprise Engineering, this is not a big problem. However, to let the Manifesto grow into the direction of a solid foundation for the Enterprise Engineering discipline as a whole, the added value of the functional perspective should be made explicit as well.

It is well possible to define functions of a system objectively and SMARTly. In areas such as mechanical engineering, that is common practice already. For example, by reflecting on the function of a vacuum bag - to trap dirt and let air through - and seeing that the industrial cyclone in a sawmill fulfills exactly the same function, 
Dyson was able to innovate the vacuum cleaner [15]. We admit that in the practice of enterprise engineering there is lots of room for improvement in defining (especially: business) functions. This is no reason however to say that it is inherently impossible to define functions objectively.

The "subjectivity", ascribed by the EEM to the function perspective, seems more to belong to the relationship of a system to its stakeholders. Indeed its stakeholder will assess the meaning, contribution and value of a certain system, against his (business and/or personal) interests and goals in that system (its results, transition, implementation or governance); one could call this subjective. It seems more adequate to explicitly address these aspects of a system in a separate perspective, named e.g. "purpose", "contribution", "goal" or, as suggested by Op "t Land et al [14], "valuation".

Using as a working definition for objective (ly) "A proposition is considered to be objectively true, when independent observers, using the same observation instruction, assert it", we can now conclude the following. In principle, both construction and function can be objectively asserted. For value, contribution and the like this is far more difficult, though not impossible. E.g., by interviewing a well chosen set of customers, one can turn the individual (subjective) customer satisfaction to a shared, inter-subjective notion of customer (or even market) satisfaction. We wonder however why the EEM introduces these - more epistemological - notions as a label überhaupt, since it uses this distinction nowhere else.

Using as a working definition for a model "a goal-oriented abstraction from reality", we can conclude the following. Each model will, by definition, be incomplete, since it consciously leaves out certain things from reality. Such - as said: inherent partiality should not be equaled to subjectivity! So the fact that a functional model of a book generally does include something about the knowledge or the story captured, and not the potential energy delivered when burned (as shown in the 2004-movie "The Day After Tomorrow") does not make this functional model subjective, though it is incomplete. To assess whether a model is adequate, it should be assessed against its goals, e.g., understanding, steering or changing an enterprise.

\subsection{Recommendations}

In the discipline of Enterprise Engineering, we propose to discern between a function perspective and a second perspective named, e.g., valuation perspective, as follows:

- a function of a system is defined as an association between a given input with exactly one output, possibly taking parameters into consideration;

- this definition of function follows common and mathematical definitions;

- one system may, and generally will, have more functions;

- a function of a system is an inherent property of the system itself;

- a commonly used metaphor or model of a function is a "machine" or "black box" that converts input into output;

- from this it follows immediately that so-called non-functional requirements are part of a functional perspective as well because they can be described as blackbox behavior of the system; 
- a valuation of a system is defined as the contribution of a function of a system for a certain stakeholder

- this (positive or negative) contribution can differ per stakeholder, because of his specific (business and/or personal) interests and goals in that system;

- one function can, and generally will, have more contributions;

- the contribution of a function of a system to a stakeholder is a property of the relationship between the system and the stakeholder.

As a consequence, we propose to change the Enterprise Engineering Manifesto as follows.

- Under "Motivation", change the statement on the value of functional knowledge to "Such (functional) knowledge is necessary and sufficient for managing an enterprise, but it is inadequate insufficient for bringing about changes".

- Split Postulate 3 in two parts $3 \mathrm{a}$ and $3 \mathrm{~b}$, in which $3 \mathrm{a}$ deals with the perspectives, and $3 \mathrm{~b}$ with corresponding models.

- Create Postulate 3a as follows: "There are three distinct perspectives on enterprises (as on all systems): construction, function and valuation. All other perspectives are a subdivision of one of these. Construction of a system deals with its operation, function deals with its behavior, and valuation with the contribution of that system for a stakeholder. Contrary to valuation, both function and construction are inherent properties of a system. Both All three perspectives are needed for developing enterprises."

- Create Postulate 3b as follows: "The function perspective of a system needs to be modeled with black-box models. Also the other way around applies: a black-box model can only be used for the function perspective. The construction perspective of a system needs to be modeled with white-box models. And also here the other way around applies: a white-box model can only be used for the construction perspective, though logic can exist which derives the function from a certain construction. Note. For convenience sake, we talk about the business of an enterprise when taking the function perspective of the customer, and about its organization when taking the construction perspective."

Other recommendations for the Enterprise Engineering Manifesto are:

- consider leaving the notions subjective / objective out of the Manifesto altogether or elaborate it, introducing inter-subjectivity as well, distinguishing between ontological and epistemological notions, and explaining the purpose of this distinction in the Manifesto;

- consider introducing explicitly the notion of model adequacy [16]; this will allow for evaluating models against its own stated goals;

- describe the whole area of Enterprise Engineering, in which function, construction (both construction on an ontological level and construction on an implementation level) and the influence of context and goals, including the influence of principles are described in a balanced and coherent way; 
- make clear the state of the art of the Enterprise Engineering discipline, giving the state of the art of the current contributing disciplines, such as management \& organization science, economics and traditional (e.g., mechanical, electrical, civil) engineering disciplines;

- first clarify the underlying vision on the EEM; is it an activistic document to correct current organization science thinking with a discipline of constructional thinking on organizations, or is the claim to broadly describe the whole area of Enterprise Engineering; we recommend the latter, but at least it should be clear.

\section{References}

1. Dietz, J.L.G.: Enterprise Engineering Manifesto (2011), http: / /www. ciaonetwork.org/publications / EEManifesto.pdf (last visited January 24, 2012)

2. Kaplan, R.S., Norton, D.P.: Strategy Maps: Converting Intangible Assets Into Tangible Outcomes. Harvard Business School Press, Boston (2004)

3. Dietz, J.L.G.: Architecture - Building strategy into design. Netherlands Architecture Forum, Academic Service - SDU, The Hague, The Netherlands (2008)

4. Dietz, J.L.G.: Enterprise Ontology: Theory and Methodology. Springer (2006)

5. Apostel, L.: Towards the formal study of models in the non-formal sciences. Synthese 12(2), 125-161 (1960)

6. Dietz, J.L.G., Hoogervorst, J.A.P.: Enterprise Ontology and Enterprise Architecture - how to let them evolve into effective complementary notions. GEAO Journal of Enterprise Architecture 1 (2007)

7. Phillips, D.C.: Subjectivity and objectivity: An objective inquiry. In: Peshkin, E.A. (ed.) Qualitative Inquiry in Education: The Continuing Debate, pp. 19-37. Teachers College Press, New York (1990)

8. Kuhn, T.S.: The Structure of Scientific Revolutions. University of Chicago Press, Chicago (1962)

9. Doran, G.T.: There's a S.M.A.R.T. way to write management's goals and objectives. Management Review, 35-36 (1981)

10. Popper, K.R.: Objective Knowledge: An Evolutionary Approach, p. 392. Oxford University Press, Oxford (1972)

11. Tsai, A.: Sprint Survey Reveals 56\% of Phone Users Do More than Talk (2006), http://www.mobiledia.com/news/43052.html (last visited February 26, 2012)

12. Skyttner, L.: General Systems Theory: Problems, Perspectives, Practice, 2nd edn. World Scientific Publishing Co. Pte. Ltd., Singapore (2005)

13. Baarda, P.J.: Smart SOA - IT Foundations and Enterprise Relativity, Via Nova Architectura (2011)

14. Op 't Land, M., Proper, H., Waage, M., Cloo, J., Steghuis, C.: Enterprise Architecture Creating Value by Informed Governance. The Enterprise Engineering. Springer, Berlin (2009)

15. Salustri, F.A.: Art Markman reinvents the wheel on innovation (2012), http: / / filsalustri.wordpress.com/2012/01/14/art-markmanreinvents-the-wheel-on-innovation/ (last visited January 21, 2012)

16. Reggiani, M.G., Marchetti, F.E.: On Assessing Model Adequacy. IEEE Transactions on Systems, Man and Cybernetics SMC-5(3), 322-330 (1975) 